\title{
Artificially ordered Bi/Sb superlattice alloys: Fabrication and transport properties
}

\author{
Sunglae Cho \\ Department of Physics, University of Ulsan, Ulsan, 680-749, South Korea
}

\begin{abstract}
Yunki Kim, Suk J. Youn, Antonio DiVenere, George K. L. Wong, ${ }^{*}$ Arthur J. Freeman, and J. B. Ketterson ${ }^{\dagger}$
Department of Physics and Astronomy, Northwestern University, Evanston, Illinois 60208

Linda J. Olafsen, ${ }^{\ddagger}$ Igor Vurgaftman, Jerry R. Meyer, and Craig A. Hoffman

Naval Research Laboratory, Code 5613, Washington, DC 20375-5338
\end{abstract}

(Received 9 April 2001; published 28 November 2001)

\begin{abstract}
We have fabricated $\mathrm{Bi} / \mathrm{Sb}$ superlattice alloys that are artificially ordered on the atomic scale using molecularbeam epitaxy. We observe that by changing the superlattice period thickness, the electronic structure can be "tuned" from a semimetal, through zero gap, to a narrow-gap semiconductor. These unique properties, which are distinct from those in random alloys, are believed to be a consequence of ordered atomic configurations.
\end{abstract}

DOI: 10.1103/PhysRevB.64.235330 PACS number(s): 61.66.Dk, 73.21. $-\mathrm{b}$, 73.50. $-\mathrm{h}$, 73.61.At

\section{INTRODUCTION}

With the help of advanced thin-film techniques, such as molecular-beam epitaxy (MBE) and metal organic chemical vapor deposition (MOCVD), artificial structures (e.g., superlattices and sandwiches) of the form $A_{m} B_{n}$ have been achieved consisting of $m$ layers of $A$ alternating with $n$ of $B$ along a specified growth direction. These artificial structures have attracted much attention as functional materials due to their interesting physical properties. It is well known that as the layer thicknesses in a film or a multilayer decreases, one can observe a size effect associated with the increased surface carrier scattering. For films with a thickness comparable to or smaller than the de Broglie wavelength of the carriers, the quantum size effect dominates and the energy spectrum of the carriers in size-quantized films breaks up into subbands, which influences the transport properties. A superlattice with constituent layer thickness of atomic size ${ }^{1}$ has been fabricated consisting of one monolayer (ML) of Fe alternated with $1 \mathrm{ML}$ of $\mathrm{Au}$ to form an "ordered alloy." The electronic structure of an artificially ordered alloy is expected to be quite different from a random alloy. ${ }^{2-4}$ Intentionally ordered $\mathrm{Si} / \mathrm{Ge}$ alloys, with individual layers of unit-cell thickness, displays a different electronic spectrum relative to a random alloy as evidenced by the production of a different phase as a consequence of the ordered atomic structure. ${ }^{5}$

In this paper we report the fabrication of short-period $\mathrm{Bi} / \mathrm{Sb}$ superlattices of atomic scale along with evidence of a changing band structure as a function of the superlattice modulation period thickness. It will be seen that even when the individual layers are $\leqslant 1$ monolayer thick, the artificial ordering is preserved and the electronic structure remains distinct from that of $\mathrm{Bi}_{1-x} \mathrm{Sb}_{x}$ random alloy with the same average Sb composition $x_{\mathrm{av}}$.

$\mathrm{Bi}$ and $\mathrm{Sb}$ are group- $\mathrm{V}$ semimetals with a rhombohedral crystal structure. They have a small energy overlap between the conduction and valence bands, small effective masses, and high mobilities. Because of these properties, Bi and Sb have frequently been used for quantum confinement studies in quantum well ${ }^{6-8}$ and quantum wire ${ }^{9-13}$ geometries. For small $x$, the addition of $\mathrm{Sb}$ to form $\mathrm{Bi}_{1-x} \mathrm{Sb}_{x}$ random alloys decreases the band overlap ${ }^{14-23}$ and results in a transition from semimetal to semiconductor when $0.07 \leqslant x \leqslant 0.22$. Above $x=0.22$ the alloy again reverts to a semimetal, since the $H$ valence band then overlaps the $L$ conduction band as it does in pure $\mathrm{Sb}(x=1)$.

The crystal structure of $\mathrm{Bi}$ and $\mathrm{Sb}$ and the atomic arrangements of both a random alloy and the artificially ordered alloy are shown in Fig. 1. Bi and $\mathrm{Sb}$ have the rhombohedral $A 7$ crystal structure, which has two atoms per unit cell located at $(u, u, u)$ and $(-u,-u,-u): \quad u=0.237$ for $\mathrm{Bi} ; 0.233$ for $\mathrm{Sb}$. The rhombohedral structure can also be described with a hexagonal unit cell containing six atoms at $\left(000 ; \frac{2}{3} \frac{1}{3} \frac{1}{3} ; \frac{1}{3} \frac{2}{3} \frac{2}{3}\right) \pm 00 u$. Along the trigonal growth axis, there are six planes making up the $11.86 \AA$ ( $\mathrm{Bi}$ ) or $11.274 \AA$ ( $\mathrm{Sb})$ unit cell, which corresponds to about $2 \AA$ for each plane. We have used MBE to form artificially ordered $\mathrm{Bi}_{m} / \mathrm{Sb}_{n}$ structures consisting of $m$ planes of Bi alternating with $n$ planes of $\mathrm{Sb}$ along the trigonal axis, where $m$ and $n$ are not necessarily integers.

\section{SAMPLE PREPARATION}

The ordered $\mathrm{Bi} / \mathrm{Sb}$ superlattice $(\mathrm{SL})$ alloys were grown on semi-insulating $\operatorname{CdTe}(111) B\left(E_{g}=1.5 \mathrm{eV}\right)$ substrates by (a)

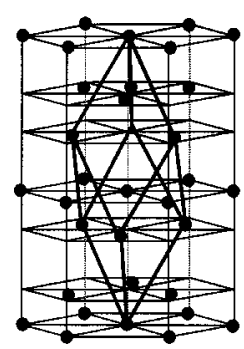

(b)

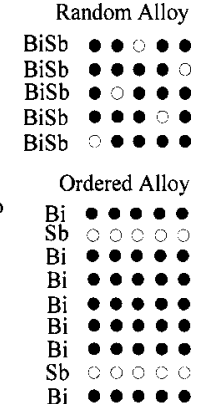

FIG. 1. (a) The crystal structure of rhombohedral Bi and $\mathrm{Sb}$. (b) The atomic arrangement of a random alloy and an artificially ordered alloy. Whereas the $\mathrm{Sb}$ atoms are randomly positioned in the random alloy, they are confined to specific planes in the ordered alloy. 


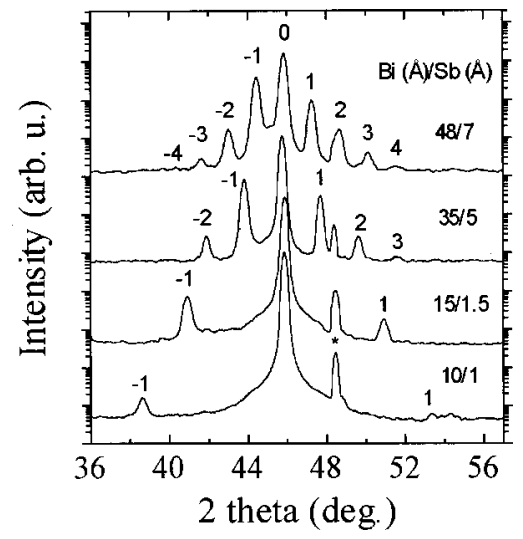

FIG. 2. $\theta-2 \theta$ x-ray scans around the (00.4) reflection for ordered $\mathrm{Bi} / \mathrm{Sb}$ superlattice alloys with different modulation periods: substrate $\mathrm{CdTe}(222)$ peak.

molecular-beam epitaxy (MBE), as described in detail elsewhere. ${ }^{23-25}$ The base pressure of the growth chamber was in the $10^{-10}$ Torr range. We first deposited a $3000-\AA$ CdTe buffer layer at $250^{\circ} \mathrm{C}$, followed by the $\mathrm{Bi}$ and $\mathrm{Sb}$ layers at a rate of about $0.4 \AA / \mathrm{s}$ and at a growth temperature of $100^{\circ} \mathrm{C}$, which was repeated to form the ordered SL. A 2-s interruption time was introduced between the deposition of $\mathrm{Bi}$ and $\mathrm{Sb}$ layers to enhance surface migration of the absorbed atoms during the growth. The lattice mismatches of $\mathrm{Bi}$ and $\mathrm{Sb}$ with the $\mathrm{CdTe}(111) \mathrm{B}$ substrate $(4.58 \AA)$ were 0.7 and $6 \%$, respectively. The growth direction of $\mathrm{Bi}$ and $\mathrm{Sb}$ layers on $\mathrm{CdTe}(111) \mathrm{B}$ is parallel to their trigonal axes. We prepared $\mathrm{Bi} / \mathrm{Sb} \mathrm{SL}$ alloys with eight different modulation periods: $7.7 \AA(7 \AA \mathrm{Bi} / 0.7 \AA \mathrm{Sb}), 11$ (10/1), 12 (10/2), 16.5 (15/1.5), $18(15 / 3), 27(24 / 3), 40(35 / 5)$, and $55(48 / 7)$, which all have average Sb compositions in the range 0.090.17 . Each alloy had a total thickness of $1.2 \mu \mathrm{m}$. The layer thicknesses were monitored by a quartz crystal microbalance. The accuracy of the composition has been confirmed by inductively coupled plasma (ICP) spectroscopy analysis. Reflection high-energy electron diffraction (RHEED) was used to examine the specific surface reconstruction during deposition of the layers.

\section{RESULTS AND DISCUSSION}

In growing SL's with sharp interfaces, two-dimensional (2D) nucleation is preferred over 3D nucleation, since the latter may introduce height variations and other defects where the 3D islands coalesce. The present in situ RHEED experiments showed a streaky pattern with Kikuchi lines ${ }^{25}$ for the $\mathrm{Bi}$ layer grown on $\mathrm{CdTe}(111) \mathrm{B}$, and also for the growth of $\mathrm{Bi}(\mathrm{Sb})$ on $\mathrm{Sb}(\mathrm{Bi})$, regardless of the $\mathrm{Bi}$ and $\mathrm{Sb}$ layer thicknesses. These observations confirm 2D layer-by-layer growth for both $\mathrm{Bi}$ on $\mathrm{Sb}$ and $\mathrm{Sb}$ on Bi. The RHEED patterns repeat every $60^{\circ}$, implying growth along the trigonal axis.

In order to verify the presence of ordering, we performed standard $\theta-2 \theta$ x-ray diffraction studies for various SL alloys. The data in Fig. 2 display a well-resolved pattern of satellite peaks. Besides the fundamental Bragg diffraction peak, the first-, second-, third-, and fourth-order SL reflections can be

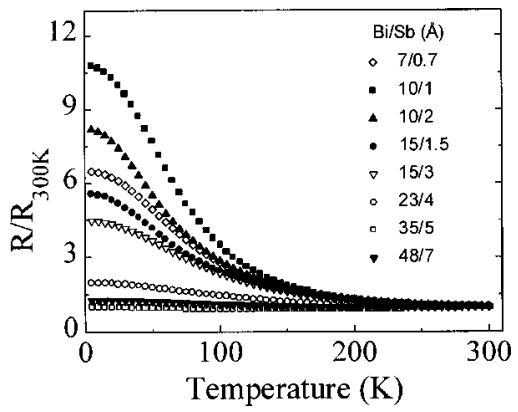

FIG. 3. Temperature-dependent electrical resistivities of ordered superlattice alloys.

seen for larger modulation periods, indicating relatively abrupt interfaces between the layers. As the modulation period decreases, the angular separation between the satellites increases. Remarkably, we see distinct satellites in SL's with monolayer and even submonolayer Sb layers: $(10 \AA \mathrm{Bi} / 1 \AA$ $\mathrm{Sb}),(10 / 2)$, and $(15 / 1.5)$. While some atomic interdiffusion is clearly unavoidable, the observation of SL satellites for structures containing monolayer and submonolayer Sb layers confirms the formation of ordered alloys with well-defined layers. Clearly, the growth temperature of only $100^{\circ} \mathrm{C}$ helps to minimize the interlayer mixing. We have earlier observed that $\mathrm{BiSb} / \mathrm{Bi}$ superlattices grown at $200^{\circ} \mathrm{C}$ did not have sharp interfaces because of severe $\mathrm{Sb}$ diffusion, whereas superlattices grown at 100 and $150{ }^{\circ} \mathrm{C}$ have sharp interfaces, as determined by $\mathrm{x}$-ray diffraction (XRD) superlattice satellites and TEM images. The angular position of the $\mathrm{x}$-ray satellite peaks yields a SL modulation period that is consistent with the value determined by the quartz crystal thickness monitor.

Figure 3 shows the temperature-dependent electrical resistivities for all of the ordered $\mathrm{Bi} / \mathrm{Sb}$ alloy films. The samples with a long period show semimetallic behavior. However, as the modulation period decreases, the electrical resistivity rapidly increases at low temperatures. This indicates that a semimetal-semiconductor transition occurs as the modulation period decreases. We have determined an effective thermal gap $\left(E_{g, \text { therm }}\right)$ for the semiconducting alloys using the empirical relation $\rho=\rho_{0} \exp \left(E_{g, \text { therm }} / 2 k_{B} T\right)$, in order to qualitatively interpret our results and compare them with the values reported for random alloys (see Ref. 23). The effective thermal energy gaps derived in this manner were 29, 29, 25, 24, and $22 \mathrm{meV}$ for the samples with modulation periods 7.7, 11, $12,16.5$, and $18 \AA$, respectively. Note that the thermal energy gaps of the SL alloys decrease with modulation period, and are smaller than the $35-40 \mathrm{meV}$ values $^{23}$ of random alloy films, also grown on $\mathrm{CdTe}(111) \mathrm{B}$, with the same average composition, but higher than the $14-20-\mathrm{meV}$ of bulk values $^{23,26}$ due to the tensile strain.

Similar qualitative behavior is observed in the temperature-dependent thermopower data shown in Fig. 4. All samples exhibit negative thermopowers because they are either $n$ type or intrinsic, and the electrons have higher mobilities than the holes (see below). The ordered alloys with modulation periods of $7.7,11,12,16.5$, and $18 \AA$ show a slight thermopower increase above $200 \mathrm{~K}$, which is characteristic of an intrinsic semiconductor. ${ }^{23}$ The magnitude of the negative thermopower increases with decreasing period, 


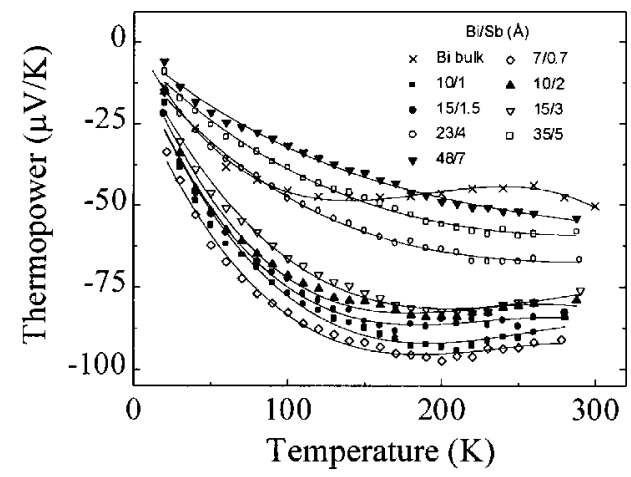

FIG. 4. Thermopower vs temperature for the ordered superlattice alloys and bulk Bi.

which also indicates an enhanced semiconducting energy gap. Other samples with longer modulation periods show semimetallic behavior, which is consistent with the electrical resistivity data. These resistivity and thermopower results provide strong evidence that the electronic structure of the ordered $\mathrm{Bi} / \mathrm{Sb} \mathrm{SL}$ alloys differs from that of random alloy films with the same thickness and average composition. Furthermore, the electronic structures can be tuned via the modulation period from semimetallic, through zero gap, to narrow band-gap semiconducting.

To investigate this phenomenon in greater detail, we carried out magnetic-field-dependent Hall and resistivity measurements on all samples at $B$ fields from 0 to $7 \mathrm{~T}$ and at 19 different temperatures between 4.2 and $300 \mathrm{~K}$. The quantitative mobility spectrum analysis (QMSA) (Refs. 27-29) technique was then applied to the data to determine effective densities and mobilities for both electrons and holes at each temperature. The results are illustrated in Fig. 5 and Table I along with earlier data ${ }^{28}$ for $\mathrm{Bi}$ and $\mathrm{Bi}_{0.91} \mathrm{Sb}_{0.09}$ films with similar thicknesses. All of the ordered SL alloys had similar low-temperature electron mobilities ranging from 7800 to $19000 \mathrm{~cm}^{2} / \mathrm{V} \mathrm{s}$, which are only slightly lower than the corresponding values for $\mathrm{Bi}$ and $\mathrm{Bi}_{0.91} \mathrm{Sb}_{0.09}$. At $300 \mathrm{~K}$, where phonon scattering dominates, all of the mobilities were

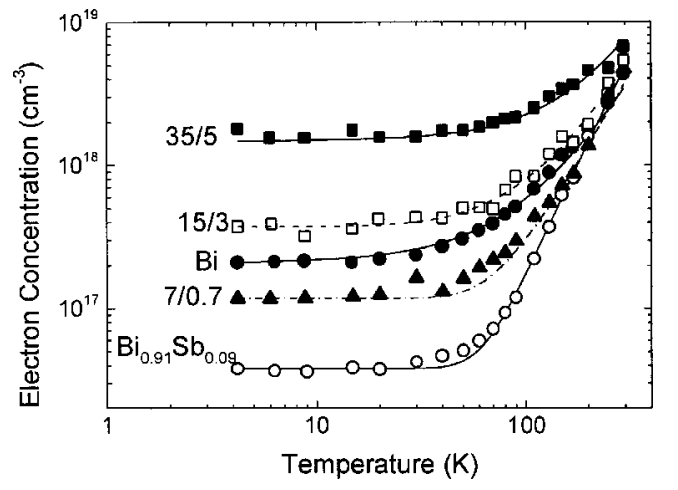

FIG. 5. Temperature-dependent electron concentrations (points) and statistical fits (curves), as determined by a QMSA of fielddependent magnetotransport measurements.

nearly identical. Low-temperature hole mobilities were generally in the $2000-5000-\mathrm{cm}^{2} / \mathrm{V}$ s range.

In the low-temperature limit, all of the ordered SL alloy samples are either $n$ type or intrinsic $(n \approx p)$, with electron concentrations in the $1.1 \times 10^{17}-1.8 \times 10^{18}-\mathrm{cm}^{-3}$ range. Figure 5 shows that the densities increase with temperature, as additional carriers are generated thermally. The curves represent fits to a statistical model, ${ }^{29}$ which incorporates the anisotropy, nonparabolicity, valley degeneracy, and a temperature dependence of the various conduction and valence band edges. The net donor concentration was fixed by the experimental values for $n_{0}-p_{0}$ in the low-temperature limit, and the band gap (either positive or negative) was used as the fitting parameter. The same empirical density-of-states (DOS) effective mass gave good fits to the high-temperature electron densities in all of the ordered SL alloy samples, although slightly different DOS masses were employed for $\mathrm{Bi}$ and $\mathrm{Bi}_{0.91} \mathrm{Sb}_{0.09}$.

The band gaps derived in this manner are listed in Table I. Because the masses and their temperature dependences are complex and not known precisely (with the possible role of $\mathrm{H}$-valley holes in the ordered SL alloys being especially uncertain), and also because the data become less sensitive to

TABLE I. Some electronic characteristics derived from a QMSA analysis of the field-dependent magnetotransport measurements. Results are given for $\mathrm{Bi}, \mathrm{a} \mathrm{Bi}_{1-x} \mathrm{Sb}_{x}$ random alloy, and ordered superlattice alloys; $n_{0}$ and $p_{0}$ are the low-temperature $(\leqslant 15 \mathrm{~K})$ concentrations of electrons and holes, respectively, and $\mu_{n}$ is the low-temperature electron mobility. All samples were either $n$ type or nominally intrinsic.

\begin{tabular}{cccccccc}
\hline \hline$L_{\mathrm{Bi}}(\AA)$ & $L_{\mathrm{Sb}}(\AA)$ & $X_{\mathrm{av}}$ & $\begin{array}{c}E_{g, \text { therm }} \\
(\mathrm{meV})\end{array}$ & $\begin{array}{c}E_{g, \text { fit }} \\
(\mathrm{meV})\end{array}$ & $n_{0}\left(\mathrm{~cm}^{-3}\right)$ & $\begin{array}{c}\mu_{n} \\
\left(\mathrm{~cm}^{2} / \mathrm{V} \mathrm{s}\right)\end{array}$ & $p_{0}\left(\mathrm{~cm}^{-3}\right)$ \\
\hline $\mathrm{Bi}_{0.91} \mathrm{Sb}_{0.09}$ & & 0.09 & 40 & 35 & $3.8 \times 10^{16}$ & $2.6 \times 10^{4}$ & $\ll n_{0}$ \\
7 & 0.7 & 0.09 & 29 & 5 & $1.2 \times 10^{17}$ & $1.9 \times 10^{4}$ & $\ll n_{0}$ \\
10 & 1 & 0.09 & 29 & 0 & $1.1 \times 10^{17}$ & $1.9 \times 10^{4}$ & $\ll n_{0}$ \\
15 & 1.5 & 0.09 & 24 & -10 & $2.2 \times 10^{17}$ & $1.9 \times 10^{4}$ & $7.0 \times 10^{16}$ \\
15 & 3 & 0.17 & 22 & -15 & $3.7 \times 10^{17}$ & $9.7 \times 10^{3}$ & $\ll n_{0}$ \\
10 & 2 & 0.017 & 25 & -20 & $3.8 \times 10^{17}$ & $7.8 \times 10^{3}$ & $\ll n_{0}$ \\
$\mathrm{Bi}$ & & 0 & & -25 & $2.1 \times 10^{17}$ & $6.0 \times 10^{4}$ & $\approx n_{0}$ \\
23 & 4 & 0.15 & & -50 & $1.3 \times 10^{18}$ & $1.6 \times 10^{4}$ & $\approx n_{0}$ \\
35 & 5 & 0.13 & & -50 & $1.8 \times 10^{18}$ & $1.8 \times 10^{4}$ & $\approx n_{0}$ \\
48 & 7 & 0.13 & & -50 & $1.5 \times 10^{18}$ & $1.4 \times 10^{4}$ & $\approx n_{0}$ \\
\hline \hline
\end{tabular}


the magnitude of the bandgap when it is very small and the nonparabolicity is strong, we should expect only limited accuracy from this procedure. For example, we obtain -25 $\mathrm{meV}$ for the band gap of $\mathrm{Bi}$, whereas more detailed experimental characterizations generally yield a value closer to $-38 \mathrm{meV}^{30}$ However, we expect the relative trends of our derived bandgaps to be meaningful and reliable.

Those trends show an unmistakable dependence of the electronic structure on the modulation period of the ordered SL alloys. It is apparent from Table I that all of the samples with thicker Sb layers $(23 / 4,35 / 5,48 / 7)$ have a much larger band overlap than pure $\mathrm{Bi}$. However, decreasing the period at fixed average $\mathrm{Sb}$ concentration leads to a progressively smaller overlap or increasing semiconductor gap. This confirms that the ordered SL's have a distinct band structure which systematically depends on layer thickness, rather than reverting to the properties of the equivalent random alloy as would occur if interdiffusion were severe. Yet it is also difficult to explain the variation of the energy gap at very small periods in terms of SL quantum confinement alone, since in the thin-layer limit the barriers become ineffective and a nonatomic model gives properties similar to those of the random alloy. The ordered SL alloys should therefore be viewed as a new kind of material.

\section{SUMMARY}

In summary, by alternating thin layers of $\mathrm{Bi}$ and $\mathrm{Sb}$ in a periodic geometry, we have fabricated $\mathrm{Bi} / \mathrm{Sb} \mathrm{SL}$ alloys that are artificially ordered on the atomic scale. X-ray data confirm that even structures with submonolayer Sb thicknesses exhibit clear periodicity. By changing the SL period thickness, the electronic structure was tuned from a semimetal, through zero gap, to a narrow-gap semiconductor. While long-period samples showed semimetallic behavior, a semimetal-semiconductor transition was observed at short periods. In treating the differences in the electronic structure between SL alloys and random alloys of similar average composition, the ordered structure should be viewed as a new kind of material.

\section{ACKNOWLEDGMENTS}

This work was supported by DARPA under Grant No. DAAG55-97-1-0130 and by the Basic Research Program of the Korea Science \& Engineering Foundation under Grant No. 2000-1-11400-002-3. Use was made of central facilities supported by the National Science Foundation, at the Materials Research Center of Northwestern University, under Award No. DMR-9120521.
*Permanent address: Physics Department, HKUST, Hong Kong, China.

${ }^{\dagger}$ Also with the Department of Electrical and Computer Engineering.

$\$$ Present address: Department of Physics and Astronomy, University of Kansas, Lawrence, KS 66045.

${ }^{1}$ Ultrathin Magnetic Structures, edited by J. A. C. Bland and B. Heinrich (Springer, Berlin, 1994), Parts I and III.

${ }^{2}$ K. Sato, E. Takeda, M. Akita, M. Yamaguchi, K. Takanashi, S. Mitani, H. Fujimori, and Y. Suzuki, J. Appl. Phys. 86, 4985 (1999).

${ }^{3}$ K. Takanashi, S. Mitani, M. Sano, H. Fujimori, H. Takajima, and A. Osawa, Appl. Phys. Lett. 67, 1016 (1995).

${ }^{4}$ S. Mitani, K. Takanashi, H. Nakajima, K. Sato, R. Schreiber, P. Grünberg, and H. Fujimori, J. Magn. Magn. Mater. 156, 7 (1996).

${ }^{5}$ T. P. Pearsall, J. Bevk, L. C. Feldman, J. M. Bonar, and J. P. Mannaerts, Phys. Rev. Lett. 58, 729 (1987).

${ }^{6}$ Yu. F. Ogrin, Pis'ma Zh. Eksp. Teor. Fiz. 3, 114 (1966) [JETP Lett. 3, 71 (1966)].

${ }^{7}$ C. A. Hoffman, J. R. Meyer, F. J. Bartoli, A. DiVenere, X. J. Yi, C. L. Hou, H. C. Wang, J. B. Ketterson, and G. K. Wong, Phys. Rev. B 48, 11431 (1993); 51, 5535 (1995).

${ }^{8}$ M. Lu, R. J. Zieve, A. van Hulst, H. M. Jaeger, T. F. Rosenbaum, and S. Radelaar, Phys. Rev. B 53, 1609 (1996).

${ }^{9}$ K. Liu, C. L. Chien, and P. C. Searson, Phys. Rev. B 58, R14 681 (1998)

${ }^{10}$ K. Liu, C. L. Chien, P. C. Pearson, and K. Yu-Zhang, Appl. Phys. Lett. 73, 1436 (1998).

${ }^{11}$ Z. Zhang, X. Sun, M. S. Dresselhaus, J. Y. Ying, and J. P. Heremans, Appl. Phys. Lett. 73, 1589 (1998).

${ }^{12}$ J. Heremans and C. M. Thrush, Phys. Rev. B 59, 12579 (1999).

${ }^{13}$ J. Heremans, C. M. Thrush, Z. Zhang, X. Sun, M. S. Dresselhaus,
J. Y. Ying, and D. T. Morelli, Phys. Rev. B 58, R10 091 (1998).

${ }^{14}$ A. L. Jain, Phys. Rev. 114, 1518 (1959).

${ }^{15}$ E. J. Tichovolski and J. G. Mavroides, Solid State Commun. 7, 927 (1969).

${ }^{16}$ G. A. Mironova, M. V. Sudakaova, and Ta. G. Ponomarev, Sov. Phys. Solid State 22, 2124 (1980).

${ }^{17}$ W. M. Yim and A. Amith, Solid-State Electron. 15, 1141 (1972).

${ }^{18}$ B. Lenoir, M. Cassart, J.-P. Michenaud, H. Scherrer, and S. Scherrer, J. Phys. Chem. Solids 57, 89 (1996).

${ }^{19}$ V. G. Alekseeva, N. F. Zaets, A. A. Kudryashov, and A. B. Ormont, Sov. Phys. Semicond. 10, 1332 (1976).

${ }^{20}$ N. B. Brandt and E. A. Svistova, J. Low Temp. Phys. 2, 1 (1970).

${ }^{21}$ E. E. Mendez, A. Misu, and M. S. Dresselhaus, Phys. Rev. B 24, 639 (1981)

${ }^{22}$ D. T. Morelli, D. L. Partin, and J. Heremans, Semicond. Sci. Technol. 5, S257 (1990).

${ }^{23}$ S. Cho, A. DiVenere, G. K. Wong, J. B. Ketterson, and J. R. Meyer, Phys. Rev. B 59, 10691 (1999).

${ }^{24}$ S. Cho, Y. Kim, A. DiVenere, G. K. Wong, J. B. Ketterson, and J. I. Hong, J. Vac. Sci. Technol. A 17, 2987 (1999).

${ }^{25}$ S. Cho, A. DiVenere, G. K. Wong, J. B. Ketterson, J. R. Meyer, and J. I. Hong, Phys. Rev. B 58, 2324 (1998).

${ }^{26}$ A. B. Shick, A. J. Freeman, and J. B. Ketterson, Phys. Rev. B 60, 15484 (1999).

${ }^{27}$ I. Vurgaftman, J. R. Meyer, C. A. Hoffman, D. Redfern, J. Antoszewski, L. Faraone, and J. R. Lindemuth, J. Appl. Phys. 84, 4966 (1998).

${ }^{28}$ I. Vurgaftman, J. R. Meyer, C. A. Hoffman, S. Cho, A. DiVenere, G. K. Wong, and J. B. Ketterson, J. Phys.: Condens. Matter 11, 5157 (1999).

${ }^{29}$ I. Vurgaftman, J. R. Meyer, C. A. Hoffman, S. Cho, J. B. Ketterson, L. Faraone, J. Antoszewski, and J. R. Lindemuth, J. Electron. Mater. 28, 548 (1999).

${ }^{30}$ R. T. Isaacson and G. A. Williams, Phys. Rev. 185, 682 (1969). 